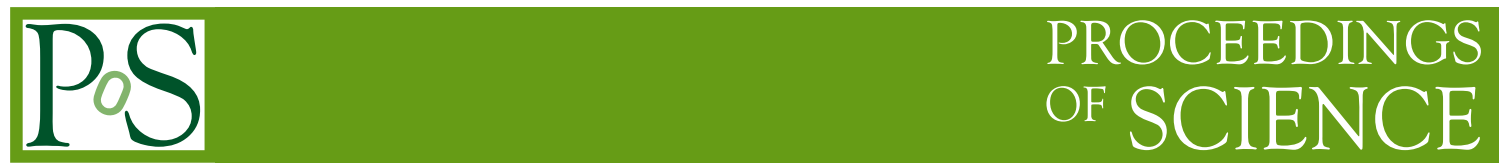

\title{
Neutron capture cross section measurements for nuclear astrophysics at n_TOF
}

Michael Heil $^{* 1}$, U. Abbondanno ${ }^{3}$, G. Aerts ${ }^{4}$, H. Álvarez-Pol ${ }^{5}$, F. Alvarez-Velarde ${ }^{6}$, S. Andriamonje ${ }^{4}$, J. Andrzejewski ${ }^{7}$, P. Assimakopoulos ${ }^{8}$, L. Audouin ${ }^{1}$, G. Badurek ${ }^{9}$, P. Baumann ${ }^{10}$, F. Bečváŕr ${ }^{11}$, E. Berthoumieux ${ }^{4}$, S. Bisterzo ${ }^{12,2}$, F. Calviño ${ }^{13}$, D. Cano-Ott ${ }^{6}$, R. Capote ${ }^{14,15}$, C. Carrapico ${ }^{16}$, P. Cennini ${ }^{17}$, V. Chepel $^{18}$, E. Chiaveri ${ }^{17}$, N. Colonna ${ }^{19}$, G. Cortes ${ }^{13}$, A. Couture ${ }^{20}$, J. Cox ${ }^{20}$, M. Dahlfors ${ }^{17}$, S. David ${ }^{21}$, I. Dillman' ${ }^{1}$, R. Dolfini ${ }^{22}$, César Domingo Pardo ${ }^{1,2}$ W. Dridi ${ }^{4}$, I. Duran ${ }^{5}$, C. Eleftheriadis ${ }^{23}$, M. Embid-Segura ${ }^{6}$, L. Ferrant ${ }^{21}$, A. Ferrari ${ }^{17}$,

R. Ferreira-Marques ${ }^{18}$, L. Fitzpatrick ${ }^{17}$, H. Frais-Koelbl ${ }^{14}$, K. Fujii ${ }^{3}$, W. Furman ${ }^{24}$, R. Gallino ${ }^{12}$, I. Goncalves ${ }^{18}$, E. Gonzalez-Romero ${ }^{6}$, A. Goverdovski ${ }^{25}$, F. Gramegna ${ }^{26}$, E. Griesmayer ${ }^{14}$, C. Guerrero ${ }^{6}$, F. Gunsing ${ }^{4}$, B. Haas ${ }^{27}$, R. Haight ${ }^{28}$, A. Herrera-Martinez ${ }^{17}$, M. Igashira ${ }^{29}$, S. Isaev ${ }^{21}$, E. Jericha ${ }^{9}$, Y. Kadi ${ }^{17}$, F. Käppeler ${ }^{1}$, D. Karamanis ${ }^{8}$, D. Karadimos ${ }^{8}$, M. Kerveno, ${ }^{10}$, V. Ketlerov ${ }^{25,17}$, P. Koehler ${ }^{30}$, V. Konovalov ${ }^{24,17}$, E. Kossionides ${ }^{31}$, M. Krtička ${ }^{11}$, C. Lamboudis ${ }^{8}$, H. Leeb ${ }^{9}$, A. Lindote ${ }^{18}$, I. Lopes ${ }^{18}$, M. Lozano ${ }^{15}$, S. Lukic ${ }^{10}$, J. Marganiec ${ }^{7}$, S. Marrone ${ }^{19}$, P. Mastinu ${ }^{26}$, A. Mengoni ${ }^{14,17}$, P.M. Milazzo ${ }^{3}$, C. Moreau ${ }^{3}$, M. Mosconi ${ }^{1}$, F. Neves ${ }^{18}$, H. Oberhummer ${ }^{9}$, M. Oshima ${ }^{32}$, S. O'Brien ${ }^{20}$, J. Pancin ${ }^{4}$, C. Papachristodoulou ${ }^{8}$, C. Papadopoulos ${ }^{33}$, C. Paradela ${ }^{5}$, N. Patronis ${ }^{8}$, A. Pavlik ${ }^{34}$, P. Pavlopoulos ${ }^{35}$, L. Perrot ${ }^{4}$, R. Plag ${ }^{1}$, A. Plompen ${ }^{36}$, A. Plukis ${ }^{4}$, A. Poch ${ }^{13}$, C. Pretel ${ }^{13}$, J. Quesada ${ }^{15}$, T. Rauscher ${ }^{37}$, R. Reifarth ${ }^{28}$, M. Rosetti ${ }^{38}$, C. Rubbia ${ }^{22}$, G. Rudolf ${ }^{10}$, P. Rullhusen ${ }^{36}$, J. Salgado ${ }^{16}$, L. Sarchiapone ${ }^{17}$, I. Savvidis ${ }^{23}$, C. Stephan ${ }^{21}$, G. Tagliente ${ }^{19}$, J.L. Tain ${ }^{2}$, L. Tassan-Got ${ }^{21}$, L. Tavora ${ }^{16}$, R. Terlizzi ${ }^{19}$, G. Vannini ${ }^{39}$, P. Vaz $^{16}$, A. Ventura ${ }^{38}$, D. Villamarin ${ }^{6}$, M. C. Vincente ${ }^{6}$, V. Vlachoudis ${ }^{17}$, R. Vlastou ${ }^{33}$, F. Voss ${ }^{1}$, S. Walter ${ }^{1}$, H. Wendler ${ }^{17}$, M. Wiescher ${ }^{20}$, K. Wisshak ${ }^{1}$

The $\mathbf{n}$-TOF Collaboration 
${ }^{1}$ Forschungszentrum Karlsruhe GmbH (FZK), Institut für Kernphysik, Germany, ${ }^{2}$ Instituto de Física Corpuscular, CSIC-Universidad de Valencia, Spain, ${ }^{3}$ Istituto Nazionale di Fisica Nucleare, Trieste, Italy, ${ }^{4}$ CEA/Saclay - DSM, Gif-sur-Yvette, France $;{ }^{5}$ Universidade de Santiago de Compostela, Spain; ${ }^{6}$ Centro de Investigaciones Energeticas Medioambientales y Technologicas, Madrid, Spain, ${ }^{7}$ University of Lodz, Lodz, Poland,${ }^{8}$ University of Ioannina, Greece ${ }^{9}$ Atominstitut der Österreichischen Universitäten,Technische Universität Wien, Austria;

${ }^{10}$ Centre National de la Recherche Scientifique/IN2P3 - IReS, Strasbourg, France $;{ }^{11}$ Charles University, Prague, Czech Republic, ${ }^{12}$ Dipartimento di Fisica Generale, Università di Torino, Italy $;{ }^{13}$ Universitat Politecnica de Catalunya, Barcelona, Spain $;{ }^{14}$ International Atomic Energy Agency, NAPC-Nuclear Data Section, Vienna, Austria $;{ }^{15}$ Universidad de Sevilla, Spain;

${ }^{16}$ Instituto Tecnológico e Nuclear(ITN), Lisbon, Portugal $;{ }^{17}$ CERN, Geneva, Switzerland; ${ }^{18}$ LIP Coimbra \& Departamento de Fisica da Universidade de Coimbra, Portugal; ${ }^{19}$ Istituto Nazionale di Fisica Nucleare, Bari, Italy $;{ }^{20}$ University of Notre Dame, Notre Dame, USA $;{ }^{21}$ Centre National de la Recherche Scientifique/IN2P3 - IPN, Orsay, France, ${ }^{22}$ Università degli Studi Pavia, Pavia, Italy; ${ }^{23}$ Aristotle University of Thessaloniki, Greece $;{ }^{24}$ Joint Institute for Nuclear Research, Frank Laboratory of Neutron Physics, Dubna, Russia, ${ }^{25}$ Institute of Physics and Power Engineering, Kaluga region, Obninsk, Russia; ${ }^{26}$ Istituto Nazionale di Fisica Nucleare(INFN), Laboratori Nazionali di Legnaro, Italy; ${ }^{27}$ Centre National de la Recherche Scientifique/IN2P3 - CENBG, Bordeaux, France, ${ }^{28}$ Los Alamos National Laboratory, New Mexico, USA, ${ }^{29}$ Tokyo Institute of Technology, Tokyo, Japan; ${ }^{30}$ Oak Ridge National Laboratory, Physics Division, Oak Ridge, USA $;{ }^{31}$ NCSR, Athens, Greece ${ }^{32}$ Japan Atomic Energy Research Institute, Tokai-mura, Japan;

${ }^{33}$ National Technical University of Athens, Greece $;{ }^{34}$ Institut für Isotopenforschung und Kernphysik, Universität Wien, Austria ${ }^{35}$ Pôle Universitaire Léonard de Vinci, Paris La Défense, France, ${ }^{36}$ CEC-JRC-IRMM, Geel, Belgium; ${ }^{37}$ Department of Physics and Astronomy - University of Basel, Basel, Switzerland, ${ }^{38}$ ENEA, Bologna, Italy, ${ }^{39}$ Dipartimento di Fisica, Università di Bologna, and Sezione INFN di Bologna, Italy.

E-mail: michael.heil@ik.fzk.de

The neutron time of flight (n_TOF) facility at CERN is a neutron spallation source with a flight path of $187.5 \mathrm{~m}$. Intense proton bunches from the CERN PS with an energy of $20 \mathrm{GeV}$, an intensity of $7 \times 10^{12}$ protons per pulse, and a pulse width of $6 \mathrm{~ns}$ are focused on a lead spallation module. The white neutron energy spectrum produced in this way ranges from thermal to several $\mathrm{GeV}$, thus covering the full energy range of interest for nuclear astrophysics, in particular for measurements of the neutron capture cross sections required in $s$-process nucleosynthesis. The combination of the long flight path of $187.5 \mathrm{~m}$, which allows to perform time-of-flight measurements with very high energy resolution in a low-background environment, with the extremely high instantaneous neutron flux and the low repetition frequency of the proton beam is quite unique and perfectly suited for measurements of neutron capture cross sections in general and on radioactive samples in particular. This contribution gives an overview of the completed neutron capture studies on isotopes of $\mathrm{Mg}, \mathrm{Zr}, \mathrm{La}, \mathrm{Sm}, \mathrm{Os}, \mathrm{Pb}$, and $\mathrm{Bi}$. Further improvements and future plans for nuclear astrophysics applications are addressed as well.

International Symposium on Nuclear Astrophysics - Nuclei in the Cosmos - IX

June 25-30 2006

CERN, Geneva, Switzerland

*Speaker. 


\section{The n_TOF facility}

The $n \_$TOF facility at CERN provides a white neutron spectrum, well suited for measurements of neutron induced reactions, in particular for capture cross section measurements relevant for $s$-process studies. Neutrons are produced by proton induced spallation in a water-cooled lead block using intense proton bunches from the CERN PS accelerator complex (momentum $20 \mathrm{GeV} / \mathrm{c}$, $7 \times 10^{12}$ protons per bunch, $6 \mathrm{~ns}$ pulse width, and a repetition rate of $0.4 \mathrm{~Hz}$ on average). About 300 neutrons are produced per proton, resulting in an unsurpassed luminosity of the neutron beam. The neutrons are slowed down in the lead block and further moderated in the $5 \mathrm{~cm}$ thick water layer surrounding the spallation module.

The long flight path of $187.5 \mathrm{~m}$ to the present measuring station enables time-of-flight measurements with excellent energy resolution in a low-background environment. Typical values for the resolution are $0.03 \%$ in the region below $100 \mathrm{eV} 0.1 \%$ at $30 \mathrm{keV}$. Neutron fluxes per energy decade are almost constant $5 \times 10^{4}$ up to $100 \mathrm{keV}$ before rising to a maximum of $2.5 \times 10^{5}$ around $1 \mathrm{MeV}$. A detailed description of the facility is given in the $\mathrm{n}_{-} \mathrm{TOF}$ performance report [1].

\section{Experiments for astrophysics}

The most important ingredients for $s$-process studies are neutron capture cross sections in the astrophysically important energy range between 0.1 and $500 \mathrm{keV}$. The $\mathrm{n}_{-} \mathrm{TOF}$ facility is perfectly suited for such measurements thanks to the broad energy spectrum, the very high luminosity, the low background environment, the very favorable duty factor. In particular, the low duty factor and the high instantaneous flux allows to perform measurements of small capture cross sections and/or the use of small sample quantities. The latter aspect is especially important for experiments which require isotopically enriched samples. In addition, the excellent energy resolution favors measurements of resonance dominated cross sections and permits to extract resonance parameters, even at higher energies, where resonances were difficult to resolve in previous experiments. The high instantaneous flux and the low duty factor are also the basis for capture measurements on radioactive samples, which are otherwise limited because of the intrinsic sample background.

Based on these features, an experimental campaign for neutron capture studies relevant for $s$-process nucleosynthesis was defined during the construction phase of the facility, which was completed in the spring of 2002. The program was focussed on three aspects: (i) Measurements of small, resonance dominated cross sections, where emphasis was put on the use of small samples in order to minimize corrections for sample effects. In this way, systematic uncertainties were well under control. Moreover, backgrounds due to sample scattered neutrons could be avoided by using especially designed $\mathrm{C}_{6} \mathrm{D}_{6}$ detectors as described below. (ii) This first set of experiments was complemented by measurements on radioactive samples, which take advantage of the high flux and the low duty factor, and by (iii) measurements on ${ }^{186,187} \mathrm{Os,} \mathrm{which} \mathrm{are} \mathrm{required} \mathrm{for} \mathrm{an} \mathrm{update} \mathrm{of} \mathrm{the}$ $\mathrm{Re} / \mathrm{Os} \mathrm{cosmic} \mathrm{clock.}$

The measurements related to the astrophysics program, which were carried out during the first two years of operation of the n_TOF facility in 2002 and 2003, are partly described in other contributions to this conference. Apart from two characteristic examples, a brief summary of the investigated reactions, of the underlying motivation, and of the present status should, therefore, 
suffice here. For more details the reader is referred to several contributions in these proceedings $[2,4,5,6]$.

- ${ }^{24,25,26} \operatorname{Mg}(n, \gamma)$

The abundance pattern of the $\mathrm{Mg}$ isotopes is expected to carry information on the strength of the $s$-process neutron source ${ }^{22} \mathrm{Ne}(\alpha, n){ }^{25} \mathrm{Mg}$, in particular with respect to the composition of presolar dust grains formed in the circumstellar layers of asymptotic giant branch (AGB) stars. The analysis of these data is still in progress.

- $90,91,92,93,94,96 \mathrm{Zr}(n, \gamma)$

The $\mathrm{Zr}$ isotopes have attracted considerable interest for the possibility to constrain the stellar neutron flux by the isotope ratio ${ }^{96} \mathrm{Zr} /{ }^{94} \mathrm{Zr}$. Due to the comparably short half-life of ${ }^{95} \mathrm{Zr}$ $\left(t_{1 / 2}=64 \mathrm{~d}\right)$ the heavier isotope can only be produced in a high neutron flux; otherwise $\beta$ decay would dominate, leaving ${ }^{96} \mathrm{Zr}$ completely outside the reaction path. On the one hand, the isotope ratio was measured in presolar dust grains and by spectroscopy of $\mathrm{ZrO}$ bands in the atmospheres of AGB stars. Comparisons with stellar model predictions were severely hampered by previous cross section uncertainties. Preliminary n_TOF results are presented in the contribution of Tagliente et al. [5] and are partly discussed in Sec. 3.

- ${ }^{139} \mathrm{La}(n, \gamma)$

Lanthanum consists almost completely of the neutron magic isotope ${ }^{139} \mathrm{La}$. Like all neutron magic isotopes, ${ }^{139}$ La exhibits a very small $(n, \gamma)$ cross section, which acts as a bottleneck for the $s$-process with the consequence that it accumulates a fairly large $s$-process abundance. Since it can be easily detected in stellar spectroscopy, lanthanum is a well suited indicator for the distribution of the $s$-process abundance component in the Galaxy. The n_TOF results [6] will contribute to a substantial improvement of this important spectroscopic tool.

- ${ }^{151} \operatorname{Sm}(n, \gamma)$

This unstable isotope represents an important branching point in the reaction path of the $s$-process that provides a test case for the temperature profile of the He burning layers in thermally pulsing low mass AGB stars. This feature results from the fact that the half-life of ${ }^{151} \mathrm{Sm}$ is reduced from the terrestrial value of $93 \mathrm{yr}$ to a few years under the typical conditions of stellar He burning due to thermal excitation of low lying nuclear states. The cross section of this isotope was the last missing information for an accurate analysis of this branching. The $n \_$TOF results $[6,7]$ are an essential part of this analysis.

- $186,187,188 \operatorname{Os}(n, \gamma)$

The interpretation of the chronometric pair ${ }^{187} \mathrm{Re} /{ }^{187} \mathrm{Os}$ as a clock for the age of the Galaxy rests on the so-called "local approximation", which means that the $s$-process abundances in certain mass regions scale inversely with the respective stellar $(n, \gamma)$ cross sections, $\mathrm{N}_{s} \times\langle\sigma\rangle$ $=$ constant. By this relation, the $s$-component of the ${ }^{187}$ Os abundance can be defined via the $\mathrm{N}_{s} \times\langle\sigma\rangle$ value of the $s$-only neighbor ${ }^{186}$ Os. Since the isotope ratio is accurately known, an accurate value of the cross section ratio is the crucial parameter for the assessment of the clock. The n_TOF measurement [4] was motivated by gaps in and discrepancies between previous data sets. 
- ${ }^{204,206,207,208} \mathrm{~Pb}(n, \gamma),{ }^{209} \operatorname{Bi}(n, \gamma)$

The reaction path of the $s$-process path ends, when the region of the $\alpha$-unstable isotopes ${ }^{210} \mathrm{Bi}$ and ${ }^{210,211} \mathrm{Po}$ are reached. The two main questions concerning this termination of the $s$-process path were the consistent description of the $\mathrm{Pb} / \mathrm{Bi}$ abundances and the role of $\alpha$ recycling. Since the cross sections of these neutron magic nuclei are among the smallest of all stable isotopes, previous data suffered from uncertain corrections for comparably massive samples as well as from considerable problems due to the neutron sensitivity of the $\gamma$-ray detectors used. Both problems were well under control in the $n \_$TOF measurements $[2,3]$.

\section{Examples: ${ }^{96} \mathrm{Zr}$ and ${ }^{151} \mathrm{Sm}$}

The capture measurements were performed with a pair of $\mathrm{C}_{6} \mathrm{D}_{6}$ scintillation detectors (Fig. 1) using the pulse height weighting technique. The samples with diameters of 10 to $20 \mathrm{~mm}$ were mounted on a target ladder together with a set of samples for evaluating the respective corrections, i.e. dummy samples for ambient and sample-related backgrounds, a gold sample for flux normalization, and samples of the neighboring isotopes for impurities in the sample material. Depending on the individual experiments, sample masses varied from $156 \mathrm{mg}\left({ }^{151} \mathrm{Sm}\right)$ to $4.9 \mathrm{~g}\left({ }^{208} \mathrm{~Pb}\right)$. In general, highly enriched samples could be used in order to keep the isotopic corrections small.

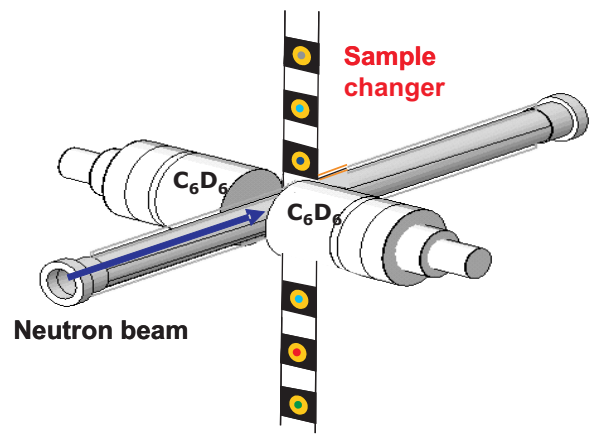

Figure 1: Setup for the n_TOF measurements of neutron capture cross sections, consisting of the evacuated beam line, a sample changer, and two $\mathrm{C}_{6} \mathrm{D}_{6}$ scintillation detectors.

In order to minimize the background from scattered neutrons, the beam line and the target ladder were fabricated from carbon fiber. Also the $\mathrm{C}_{6} \mathrm{D}_{6}$ detectors were optimized with respect to neutron sensitivity [8]. The remaining contribution from scattered neutrons was measured with a carbon scattering sample or by using black-resonance filters, which eliminate neutrons at specific energies from the beam so that the background level can be determined from the measured spectra.

The spectra of the capture yields shown in Fig. 2 correspond to examples, where the cross sections in the astrophysically relevant keV-region exhibit pronounced resonance structures $\left({ }^{96} \mathrm{Zr}\right)$ or a smooth energy dependence $\left({ }^{151} \mathrm{Sm}\right)$. While the signal-to-background ratio in the latter case is fairly large, one finds that backgrounds can be crucial for the analysis of the resonance-dominated spectrum of ${ }^{96} \mathrm{Zr}$. In the keV-region, the main background contribution is due to in-beam photons, which originate from neutron captures on hydrogen in the water moderator and are then scattered in the sample. This component could be considerably reduced by placing the $\mathrm{C}_{6} \mathrm{D}_{6}$ detectors 10 $\mathrm{cm}$ upstream of the samples at backward angles of $\approx 125^{\circ}$ with respect to the neutron beam. The 
remaining background was determined with a lead sample, which has a low neutron capture and a high photon scattering cross section.
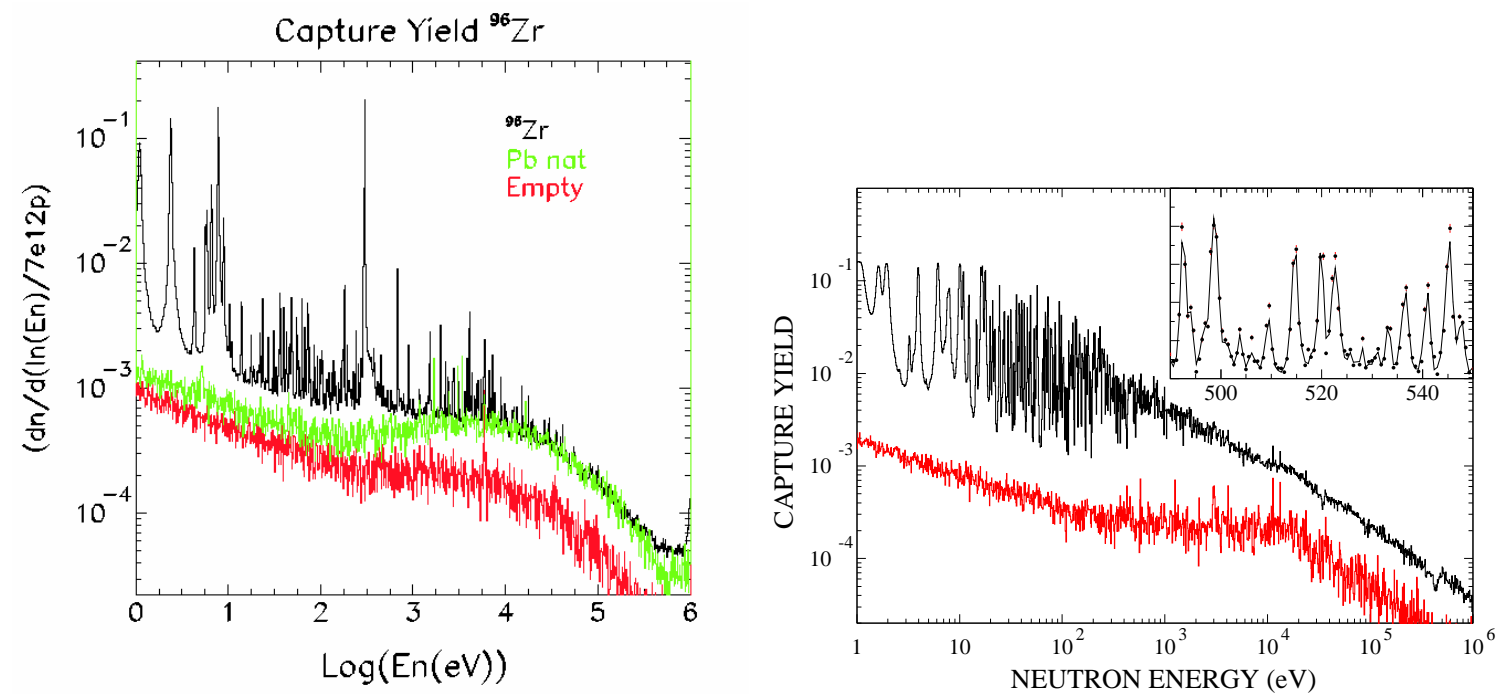

Figure 2: Measured capture yields of the ${ }^{96} \mathrm{Zr}(\mathrm{n}, \gamma)$ (left panel) and ${ }^{151} \mathrm{Sm}$ reactions (right panel) in the neutron energy range $1 \mathrm{eV}-1 \mathrm{MeV}$. The ambient background was measured with an empty position in the sample ladder. The main background contribution in the $\mathrm{keV}$ region is due to in-beam photons scattered by the sample. This component, which is crucial for the resonance dominated cross section of ${ }^{96} \mathrm{Zr}$, can be measured by means of a lead sample.

The setup sketched in Fig. 1 has been complemented in 2004 by a total absorption calorimeter consisting of 40 large $\mathrm{BaF}_{2}$ crystals, which form a spherical shell $15 \mathrm{~cm}$ in thickness around the capture sample. For reasons of compatibility, the concept of this array was adopted from the Karlsruhe $4 \pi \mathrm{BaF}_{2}$ detector [9] with several improvements for reducing the neutron sensitivity.

\section{Future activities}

After the successful operation of the n_TOF facility in the years 2002-2004 a new measuring campaign will be launched in 2007. At that time, a new cladded $\mathrm{Pb}$ spallation target will be installed with different options for the moderator. Measurements can then be performed with normal water as before, but in many cases the use of heavy water will be of great advantage, because (i) the intensity of the in-beam $\gamma$-background will be practically negligible and (ii) the neutron flux in the relevant keV region will be about five times higher. The price to be paid for these improvements will be a slightly reduced resolution in neutron energy, which, however, has a minor impact on $s$-process related measurements. The design of the new spallation target will be made such that it is also suited for a second, vertical flight path of about $20 \mathrm{~m}$, which is presently under discussion, and which would provide an increase in sensitivity by two to three orders of magnitude.

The astrophysics program for the near future is summarized in Table 1. It is foreseen to concentrate on neutron capture measurements on isotopes around the Fe-peak elements, which are particularly important for the weak $s$ process in massive stars, and on intermediate mass nuclei, where such data are needed for the interpretation of isotope pattern in presolar dust grains (see 
Table 1: Program in near future

\begin{tabular}{|c|c|}
\hline $\begin{array}{l}(n, \gamma) \text { measurements on } \\
\mathrm{Mo}, \mathrm{Ru}, \text { and } \mathrm{Pd} \text { isotopes }\end{array}$ & $\begin{array}{l}\text { Decomposition of } s \text { - and } r \text {-process components for determining more } \\
\text { accurate } r \text { residuals and analysis of isotopic patterns in } \mathrm{SiC} \text { grains }\end{array}$ \\
\hline $\begin{array}{l}(n, \gamma) \text { measurements on } \\
\mathrm{Fe}, \mathrm{Ni}, \mathrm{Zn} \text {, and } \mathrm{Se} \text { isotopes } \\
\text { (including }{ }^{63} \mathrm{Ni} \text { and }{ }^{79} \mathrm{Se} \text { ) }\end{array}$ & $\begin{array}{l}\text { Quantitative description of } s \text {-process nucleosynthesis in massive } \\
\text { stars needed for weak } s \text { process early contributions to chemical } \\
\text { enrichment of galactic material }\end{array}$ \\
\hline $\begin{array}{l}(n, \gamma) \text { measurements in } \\
\text { the } \mathrm{A} \approx 150 \text { region }\end{array}$ & $\begin{array}{l}\text { Improved analyses of the important s-process branchings in the rare } \\
\text { earth region between Ce and Gd, which are best suited for testing } \\
\text { stellar evolution during the AGB phase }\end{array}$ \\
\hline $\begin{array}{l}(n, \alpha) \text { measurements on } \\
{ }^{147} \mathrm{Sm},{ }^{67} \mathrm{Zn},{ }^{99} \mathrm{Ru} \\
\text { and }{ }^{58} \mathrm{Ni}(n, p)\end{array}$ & $\begin{array}{l}\text { Most of the reaction rates for describing explosive nucleosynthesis } \\
\text { in the so-called } p \text { process are being derived by statistical model } \\
\text { calulations; the input to these calculations - and in particular the } \\
\alpha \text {-nucleus optical potential, have to improved by means of } \\
\text { experimental data covering a wide mass range }\end{array}$ \\
\hline
\end{tabular}

contributions by A. Davis and E. Zinner in these proceedings) as well as for a more accurate decomposition of the $s$ - and $r$-process components. In both fields, existing cross section data are inconsistent and too uncertain for meaningful abundance calculations. Furthermore, it is planned to extend the astrophysical program by studies of $(n, \alpha)$ and $(n, p)$ cross sections on various nuclei of relevance for the $p$ process that takes place during supernova explosions.

\section{References}

[1] U. Abbondanno et al. (The n_TOF Collaboration), Report CERN-SL-2002-053, CERN, (2003). Available online on: www.cern.ch/n TOF.

[2] C. Domingo-Pardo et al. (The n_TOF Collaboration), these proceedings.

[3] C. Domingo-Pardo et al., (The n_TOF Collaboration), Phys. Rev. C (in press).

[4] M. Mosconi et al. (The n_TOF Collaboration), these proceedings.

[5] G. Tagliente et al. (The n_TOF Collaboration), these proceedings.

[6] S. Marrone et al. (The n_TOF Collaboration), these proceedings.

[7] S. Marrone et al., (The n_TOF Collaboration), Phys. Rev. C 73, 034604 (2006).

[8] R. Plag et al., Nuclear Instruments and Methods in Physics Research A 496, 425 (2003).

[9] K. Wisshak, K. et al., Nucl. Instr. Meth. A 292, 595 (1990). 